The Impact of Large-Scale Surveys on Pulsating Star Research ASP Conference Series, Vol. 203, 2000

L. Szabados \& D. W. Kurtz, eds.

\title{
A Photometric Survey for Variable Stars in Twelve Metal-Rich Globular Clusters
}

\author{
Andrew C. Layden ${ }^{1}$, Benjamin T. Bowes ${ }^{2}$, Laura A. Ritter ${ }^{2}$, \\ Douglas L. Welch ${ }^{3}$, Tracy M. A. Webb ${ }^{3}$
}

\begin{abstract}
We describe a photometric survey for variable stars in twelve metal-rich globular clusters, and provide results for the first two clusters.
\end{abstract}

The census of variable stars in metal-rich globular clusters $([\mathrm{Fe} / \mathrm{H}]>-0.8$ dex) is badly incomplete. Most of the clusters have not been searched to the depth of the horizontal branch. This hinders comparisons of the formation rate and mechanisms of cluster $R R$ Lyrae variables with the more frequently occurring field RR Lyrae stars. RR Lyrae stars in and toward the clusters also provide accurate estimates of the reddening toward these low-latitude objects.

To remedy this situation, we obtained time series observations in the $V$ and $I$ passbands for each of the twelve target clusters. We used the $0.9-\mathrm{m}$ telescope at the Cerro Tololo Inter-American Observatory and a large-format CCD. We chose the exposure times to ensure good photometry of the long period variables on the red giant branch and of the RR Lyrae variables.

We reported the results for our first cluster, NGC 6441, in Layden et al. (1999). The results for our second cluster, NGC 6316, are as follows. The color-magnitude diagram displays the red clump morphology of a typical metalrich globular cluster. We detected about 20 long-period variable stars (seven probable cluster members), two eclipsing binaries (foreground), and at least 6 RR Lyrae variables. All of the RR Lyrae variables appear to lie in the foreground, with the possible exception B32, which has the correct color and magnitude to be a cluster member. B32 also lies close to the cluster center. However, our best period estimate for B32 is $2.075 \mathrm{~d}$, suggestive of an Anomalous or Type II Cepheid lying behind the cluster. More observations are required to confirm this period. Using the apparent colors of three foreground RRab stars, we determined the reddening to be $E(B-V)=0.62 \pm 0.03 \mathrm{mag}$ (following the method of Mateo et al. 1995).

\section{References}

Layden, A. C., Ritter, L. A., Welch, D. L., \& Webb, T. M. A. 1999, AJ, 117, 1313

Mateo, M., Udalski, A., Szymański, M., et al. 1995, AJ, 109, 588

\footnotetext{
${ }^{1}$ Bowling Green State University, Bowling Green, OH 43403, U.S.A.

${ }^{2}$ University of Michigan, Ann Arbor, MI 48109, U.S.A.

${ }^{3}$ McMaster University, Hamilton, ON L8S 4M1, Canada
} 